\title{
Joint Pricing and Inventory Control with a Markovian Demand Model
}

\author{
Rui Yin \\ Kumar Rajaram \\ UCLA Anderson School of Management \\ 110 Westwood Plaza \\ Los Angeles, CA 90095 \\ Email: rui.yin@anderson.ucla.edu \\ kumar.rajaram@anderson.ucla.edu
}

October 13, 2005

\begin{abstract}
We consider the joint pricing and inventory control problem for a single product with a finite horizon and periodic review. The demand distribution in each period is determined by an exogenous Markov chain. Pricing and ordering decisions are made at the beginning of each period and all shortages are backlogged. The surplus costs as well as fixed and variable costs are state dependent. We show the existence of an optimal $(s, S, p)$-type feedback policy for the additive demand model. We extend the model to the case of emergency orders and also incorporate capacity and service level constraints. We compute the optimal policy for a class of Markovian demand and illustrate the benefits of dynamic pricing over fixed pricing strategies through numerical examples. The results indicate that it is more beneficial to implement the dynamic pricing strategy in a Markovian demand environment with a high fixed ordering cost or with high demand uncertainty.
\end{abstract}

Keywords: joint pricing and inventory control, Markovian demand, optimal feedback policy 


\section{Introduction}

The joint pricing and inventory control problem has been studied by many researchers in the operations management literature, starting with the work of Whitin (1955). The basic idea is to integrate the pricing decision with the replenishment policy when managing product inventory. In this problem, retailers act as price setters and can adjust prices dynamically to influence demand and potentially gain higher profits. Other well-known examples in the service industry are found in revenue management, which has been adopted by all major airlines, many hotel chains and car rental companies. (Kimes 1989).

Most of the recent papers that address the pricing and inventory control coordination problem with periodic review assume that demand in different periods are independent random variables. In practice, demand usually fluctuates and depends on many exogenous factors such as economic conditions, natural disasters, strikes, etc. In addition, when a competitor introduces a new product to the market, some customers may switch to the new product and consequently, the retailer's average demand may drop dramatically during some periods. In these cases, a "state-dependent" demand model seems to be more appropriate to capture such randomly changing environmental factors. Furthermore, if demand is highly price-sensitive, retailers could combine pricing decisions with replenishment planning and use price as an effective tool to hedge against demand uncertainty. Therefore there is a need to consider the joint pricing and inventory control problem in a fluctuating demand environment, and a Markovian demand modeling approach provides an effective mechanism to address this problem.

The purpose of this paper is to characterize the structure of the optimal replenishment and pricing decisions with a Markovian demand model, and to illustrate the benefits of dynamic pricing strategies through numerical examples. Specifically, we consider a single product, periodic review system with a finite horizon, where demand is price-dependent and its distribution 
at each time period is determined by an exogenous Markov chain. The ordering cost consists of a fixed cost and a variable cost, and all the cost parameters are state and time dependent. Under the assumptions of an additive demand function and full backlogging, we establish the structure of an optimal Markov (feedback) policy. We also present an algorithm to compute and analyze this policy.

There are two streams of literature that are related to our paper. The first stream is the coordination of pricing and inventory control with independent demand, as mentioned above. In this stream of research, demand is a random variable that depends on price. Under the assumption that unsatisfied demand in each period is fully backlogged, Federgruen and Heching (1999) and Chen and Simchi-Levi (2004a, 2004b) have considered periodic review models with both finite and infinite horizons. In Federgruen and Heching (1999), the ordering cost is proportional to the order quantity, and there is no setup cost. They prove a base-stock list price policy is optimal. In this policy, the optimal replenishment policy in each period is characterized by an order-up-to level, and the optimal price depends on the initial inventory level at the beginning of the period. Furthermore, the optimal price is a nondecreasing function of the initial inventory level. Chen and Simchi-Levi (2004a, 2004b) include a fixed ordering cost in their models. They prove an $(s, S, p)$-type policy is optimal for the finite horizon model with additive demand, and a stationary $(s, S, p)$ policy is optimal for the discounted and average profit models with general demand functions in the infinite horizon problem. In such a policy, the period inventory is managed using the classical $(s, S)$ policy, and the optimal price depends on the inventory position at the beginning of the period. Feng and Chen (2004) consider a long-run average profit model with periodic review and an infinite horizon. The optimality of an $(s, S, p)$-type policy is also established.

When unsatisfied demands are assumed to be lost, Polatoglu and Sahin (2000) characterize the form of the optimal replenishment policy under a general price-demand relationship and 
provide a sufficient condition for it to be of the $(s, S)$-type. For a finite horizon system, Chen, Ray and Song (2003) and Huh and Janakiraman (2005) have proved the optimality of an $(s, S, p)$ policy under assumptions of stationary parameters and a salvage value that is equal to the unit purchasing cost. In the area of continuous review models, Feng and Chen (2003) assume demand follows a Poisson process with price-sensitive intensities, while Chen, Wu and Yao (2004) model the demand process as a Brownian motion with a drift rate that is a function of price. Furthermore, in terms of techniques to prove the optimality of an $(s, S, p)$-type policy, Chen and Simchi-Levi (2004a), and Chen, Ray and Song (2003) use induction and the dynamic programming formulations, which are similar to that in Scarf (1960) in the classic stochastic inventory control problem. Huh and Janakiraman (2005) propose an alternative approach for the optimality proof, which is based on the method used in Veinott (1966). For a review of other work in the pricing and inventory literature, the reader is referred to Petruzzi and Dada (1999), Elmaghraby and Keskinocak (2003) and Chan et al. (2004).

The second stream of related literature is the inventory control problem with a Markovian demand model. Song and Zipkin (1993) present a Markovian-modulated model to capture the fluctuating demand environment. Specifically, they assume that demand in each period follows a Poisson process whose rate depends on the demand state. Sethi and Cheng (1997) analyze a general finite horizon inventory model with a Markovian demand process. They show that under certain technical assumptions, the optimal policy for the finite horizon problem is still of $(s, S)$ type, with $s$ and $S$ dependent on the demand state and the time remaining. Cheng and Sethi (1999) extend their previous work to the lost sales case and establish the optimality of $(s, S)$-type policies based on certain weak conditions on the holding, shortage and unit ordering costs. Another paper that is related to ours is Beyer, Sethi and Taksar (1998), which establishes the existence and verification theorems of an optimal feedback policy.

Recently, Gayon et al. (2004) consider a Markov Modulated Poisson Process that is similar 
to Song and Zipkin (1993), except that the fluctuating intensities are functions of price. The unit ordering cost is given and there is no fixed ordering cost. All the shortages are lost. They generalize some structural results in Li (1998) and prove an optimal base-stock policy exists for the discounted infinite horizon Markov decision process. The base-stock policy is similar to that in Federgruen and Heching (1999).

To the best of our knowledge, this is the first paper in the literature to address the joint pricing and inventory control problem with a Markovian demand in a periodic-review system and a fixed ordering cost. This paper makes the following contributions. First, under the assumptions of an additive demand function and full backlogging, we establish the optimality of a feedback policy of $(s, S, p)$-type. Second, we extend the basic model to the case when the unsatisfied demand at the end of a period is filled by an emergency order. Under certain practical assumptions on the holding cost, the regular and emergency ordering cost functions, we prove the state-dependent $(s, S, p)$ policy is still optimal for the case with additive demand. Third, we provide another extension by incorporating the storage and service level constraints to the basic model. Fourth, we develop an algorithm to compute the optimal policy for a class of Markovian demand with an arbitrary probability transition matrix and discrete, uniformly distributed random noise. Finally, we use this algorithm to illustrate the benefits of dynamic pricing over the fixed pricing strategies through extensive numerical examples. The results indicate that it is more beneficial to implement the dynamic pricing strategy in a Markovian demand environment with a high fixed ordering cost or with high demand uncertainty.

This paper is organized as follows. In Section 2, we introduce the notations and assumptions used in this paper and develop a general finite horizon inventory model with a Markovian demand process. In Section 3, we state the dynamic programming equations for the problem and establish the existence of an optimal feedback policy. In Section 4, the additive demand function is analyzed and the optimality of an state-dependent $(s, S, p)$ policy is proved. Two extensions 
of the basic model are presented in Section 5. In Section 6, we discuss the computation of the optimal policy for a class of Markovian demand and present numerical examples to illustrate the benefits of dynamic pricing over fixed pricing. Section 7 concludes the paper and presents future research directions.

\section{Model Formulation}

Consider a firm that has to make production and pricing decisions simultaneously at the beginning of every period over a finite time horizon with $N$ periods. The demand distribution at each period is determined by an exogenous Markov chain. In order to specify the pricing and inventory control problem, we introduce the following notations:

$$
\begin{aligned}
<0, N> & =\langle 0,1,2, \cdots, N>\text {, the horizon of the problem; } \\
I & =\{1,2, \cdots, L\}, \text { a finite collection of possible demand states; } \\
i_{k} & =\text { the demand state in period } k ; \\
\left\{i_{k}\right\} & =\text { a Markov chain with the }(L \times L) \text {-transition matrix } P=\left(p_{i j}\right) ; \\
\xi_{k} & =\text { demand at the end of period } k, k=0,1, \cdots, N-1 ; \\
p_{k} & =\text { selling price in period } k ; \\
p_{k} & =\text { the lower bound on } p_{k} ; \\
p_{k} & =\text { the upper bound on } p_{k} ; \\
u_{k} & =\text { the non-negative order quantity in period } k ; \\
x_{k} & =\text { the surplus (inventory/backlog) level at the beginning of period } k \text { before the ordering; } \\
y_{k} & =\text { the inventory position at the beginning of period } k \text { after the ordering; }
\end{aligned}
$$


Throughout this paper, we assume that demand $\xi_{k} \geq 0$ and $\xi_{k}$ depends on the demand state $i_{k}$. Specifically, when demand is in state $i \in I$, and the selling price is $p$, the demand functions have the following additive forms:

$$
\xi_{k}^{i}=D_{k}(i, p)+\beta_{k}^{i}
$$

where $D_{k}(i, p)$ is the non-negative, strictly decreasing deterministic or riskless demand function. We assume that this is a continuous function of $p . \beta_{k}^{i}$ is the only random component and we assume it is independent of the price $p$. Note (1) is a direct translation of the demand function in Chen and Simchi-Levi (2004a). For example, when demand is in state $i$, one commonly uses the riskless linear demand function $D_{k}(i, p)=a_{k}^{i}-b_{k}^{i} p$ for $p \leq a_{k}^{i} / b_{k}^{i},\left(a_{k}^{i}, b_{k}^{i}>0\right)$. We also assume that when $i_{k}=i, \beta_{k}^{i}$ is distributed over the interval of $\left[t_{1}, t_{2}\right]$ with the density function $\phi_{i, k}(\cdot)$. Without loss of generality, we assume that $E\left(\beta_{k}^{i}\right)=0$ and the probability of negative demand is zero.

Notice that when the price is $p$, the expected demand in period $k$ given $i_{k}=i$ is:

$$
E\left(D_{k}(i, p)+\beta_{k}^{i}\right)=D_{k}(i, p)+E\left(\beta_{k}^{i}\right)=D_{k}(i, p)
$$

We assume that the expected demand is finite for every $p \in\left[\underline{p}_{k}, \bar{p}_{k}\right]$. Since $D_{k}(i, p)$ is a strictly decreasing function of $p$, there is a one-to-one correspondence between the price and the expected demand. Also, when the firm charges price $p$ in period $k$, the expected revenue given $i_{k}=i$ is:

$$
R_{k}(i, p)=E\left(\left(D_{k}(i, p)+\beta_{k}^{i}\right) p\right)=D_{k}(i, p) p
$$

We make the following assumption on the expected revenue functions, which is similar to Chen and Simchi-Levi (2004a). This assumption is used in the discussion of preliminary results in Section 3 and in the proof of Theorem 3. 
Assumption 1. For all $k, k=0,1, \cdots, N-1$, the expected revenue in period $k$ given demand state $i_{k}=i, i \in I, R_{k}(i, p)$, is a concave function of the price $p$.

At the beginning of period $k$, an order $u_{k} \geq 0$ is placed with the knowledge that the demand state is $i_{k}$ and it will be delivered at the end of period $k$, but before the demand is realized. We assume that unsatisfied demand is fully backlogged. Thus the model dynamics can be expressed as:

$$
\left\{\begin{array}{l}
x_{k+1}=x_{k}+u_{k}-\xi_{k}^{i_{k}}, k=n, \cdots, N-1, \\
x_{n}=x \\
i_{k}, k=n, \cdots, N-1, \text { follows a Markov chain with transition matrix } P, \\
i_{n}=i .
\end{array}\right.
$$

Equation (2) describes the dynamics from period $n$ onward, given the initial inventory level $x$ and the demand state $i$.

For each period $k=0,1, \cdots, N-1$, and demand state $i \in I$, we define the following costs:

(a) $c_{k}(i, u)=K_{k}^{i} \delta(u)+c_{k}^{i} u$, the cost of ordering $u \geq 0$ units in period $k$ when $i_{k}=i$, where the fixed ordering $\operatorname{cost} K_{k}^{i} \geq 0$ and the variable $\operatorname{cost} c_{k}^{i}$ are also state dependent.

(b) $f_{k}(i, x)$, the surplus cost when $i_{k}=i$ and $x_{k}=x$. We assume $f_{k}$ is convex in $x$ and there exists $\bar{f}>0$, such that $f_{k}(i, x) \leq \bar{f}(1+|x|)$.

(c) $f_{N}(i, x)$, the penalty or disposal cost for the terminal surplus. We assume $f_{N}$ is convex in $x$ with $f_{N}(i, x) \leq \bar{f}(1+|x|)$.

The objective of our model is to decide on ordering and pricing policies in order to maximize total expected profit over the entire planning horizon. Thus, given $i_{n}=i$ and $x_{n}=x$, the objective function to be maximized during the interval $\langle n, N\rangle$ is:

$$
J_{n}(i, x ; U)=E\left\{\sum_{k=n}^{N-1}\left[p_{k} \xi_{k}^{i_{k}}-c_{k}\left(i_{k}, u_{k}\right)-f_{k}\left(i_{k}, x_{k}\right)\right]-f_{N}\left(i_{N}, x_{N}\right)\right\}
$$


where $U=\left(u_{n}, p_{n}, \cdots, u_{N-1}, p_{N-1}\right)$ is a history-dependent admissible decision for the problem.

Define the value function for the problem over the interval $\left\langle n, N>\right.$ with $x_{n}=x$ and $i_{n}=i$ to be:

$$
v_{n}(i, x)=\sup _{U \in \mathcal{U}} J_{n}(i, x ; U)
$$

where $\mathcal{U}$ denotes the class of all admissible decisions.

The objective function (3) is slightly different from the one used in Chen and Simchi-Levi (2004a). We assume the surplus costs $f_{k}\left(i_{k}, x_{k}\right)$ are charged at the beginning of the periods as in Sethi and Cheng (1997), while Chen and Simchi-Levi (2004a) and most other literature use $f_{k}\left(i_{k}, x_{k+1}\right)$. Note these two formulations are essentially similar, since $x_{k+1}$ is also the ending inventory of period $k$. Furthermore, when we start with zero initial inventory at the beginning of the entire horizon, the difference between these two formulations is even smaller. It also turns out that in the emergency order case that we will discuss in Section 5, our formulation would be more convenient for the analysis. Thus to keep consistent notations with the emergency order case, we will charge the surplus cost at the beginning of every period.

\section{$3 \quad$ Preliminary Results}

Using the principle of optimality, we can write the following dynamic programming equations for the value function. For $n=0,1, \cdots, N-1$ and $i \in I$,

$$
\begin{aligned}
v_{n}(i, x) & =-f_{n}(i, x)+\sup _{u \geq 0, \bar{p}_{n} \geq p \geq \underline{p}_{n}}\left\{R_{n}(i, p)-c_{n}(i, u)+E\left[v_{n+1}\left(i_{n+1}, x+u-\xi_{n}^{i_{n}}\right) \mid i_{n}=i\right]\right\} \\
& =-f_{n}(i, x)+c_{n}^{i} x+G_{n}(i, x)
\end{aligned}
$$


where

$$
\begin{aligned}
G_{n}(i, x) & =\sup _{y \geq x, \bar{p}_{n} \geq p \geq \underline{p}_{n}}\left[-K_{n}^{i} \delta(y-x)+g_{n}(i, y, p)\right], \text { and } \\
g_{n}(i, y, p) & =R_{n}(i, p)-c_{n}^{i} y+E\left[v_{n+1}\left(i_{n+1}, y-D_{n}\left(i_{n}, p\right)-\beta_{n}^{i_{n}}\right) \mid i_{n}=i\right] .
\end{aligned}
$$

Clearly, $v_{N}(i, x)=-f_{N}(i, x)$.

Let $B_{0}$ denote the class of all continuous functions from $I \times R$ into $R^{+}$and the pointwise limits of sequences of these functions (Feller 1971), where $R=(-\infty, \infty)$ and $R^{+}=[0, \infty)$. Note that this includes upper semicontinuous functions. Let $B_{1}$ be the subspace of functions in $B_{0}$ that are of linear growth, i.e., for any $b \in B_{1}, 0 \leq b(i, x) \leq C_{b}(1+|x|)$ for some $C_{b}>0$. Let $B_{2}$ be the subspace of functions in $B_{1}$ that are upper semicontinuous. Then for any $b \in B_{1}$, define:

$$
\begin{aligned}
F_{n+1}(b)(i, z) & =E\left[b\left(i_{n+1}, z-\beta_{n}^{i_{n}}\right) \mid i_{n}=i\right] \\
& =\sum_{j=1}^{L} p_{i j} \int_{t_{1}}^{t_{2}} b(j, z-t) \phi_{i, n}(t) d t
\end{aligned}
$$

By Lemma 2.1 in Beyer, Sethi and Taksar (1998), $F_{n+1}$ is a continuous linear operator from $B_{1}$ into $B_{1}$. Thus if $v_{n+1}(i, x)$ is continuous in $x$, then $E\left[v_{n+1}\left(i_{n+1}, y-D_{n}\left(i_{n}, p\right)-\beta_{n}^{i_{n}}\right) \mid i_{n}=\right.$ $i]=F_{n+1}\left(v_{n+1}\right)\left(i, y-D_{n}\left(i_{n}, p\right)\right)$ is jointly continuous in $(y, p)$ since $y-D_{n}(i, p)$ is continuous in $(y, p)$. From $(7)$, we know that $g_{n}(i, y, p)$ is jointly continuous in $(y, p)$. Therefore $-K_{n}^{i} \delta(y-$ $x)+g_{n}(i, y, p)$ is upper semicontinuous in $(y, p)$ and its maximum over a compact set is attained. Specifically, for any $y \geq x$, there exists $p_{n}(i, y) \in\left[\underline{p}_{n}, \bar{p}_{n}\right]$, such that

$$
\begin{aligned}
G_{n}(i, x) & =\sup _{y \geq x} \sup _{\bar{p}_{n} \geq p \geq \underline{p}_{n}}\left[-K_{n}^{i} \delta(y-x)+g_{n}(i, y, p)\right] \\
& =\sup _{y \geq x}\left[-K_{n}^{i} \delta(y-x)+g_{n}\left(i, y, p_{n}(i, y)\right)\right]
\end{aligned}
$$


where

$$
g_{n}\left(i, y, p_{n}(i, y)\right)=\max _{\bar{p}_{n} \geq p \geq \underline{p}_{n}} g_{n}(i, y, p)=R_{n}\left(i, p_{n}(i, y)\right)-c_{n}^{i} y+F_{n+1}\left(v_{n+1}\right)\left(i, y-D_{n}\left(i, p_{n}(i, y)\right)\right),
$$

and $g_{n}\left(i, y, p_{n}(i, y)\right)$ is continuous in $y$. In view of Proposition 4.2 in Sethi and Cheng (1997) with $A=-\infty$ and $B=\infty$, we know that $G_{n}(i, x)$ is continuous in $x$. Therefore $v_{n}(i, x)$ is continuous in $x$ and the original dynamic programming equation (5) can be rewritten as

$$
\begin{aligned}
& v_{n}(i, x)=-f_{n}(i, x)+c_{n}^{i} x+\sup _{y \geq x}\left[-K_{n}^{i} \delta(y-x)+g_{n}\left(i, y, p_{n}(i, y)\right)\right], \text { and } \\
& v_{N}(i, x)=-f_{N}(i, x) .
\end{aligned}
$$

From (10) and (11), the original two-variable, joint optimization problem is transformed to the traditional periodic review inventory problem with price given. Therefore we are only left to determine the replenishment policy. Next, we present two verification theorems similar to Theorems 2.1 and 2.2 in Beyer, Sethi and Taksar (1998), which establish the existence of an optimal feedback policy. We need the following assumption on the cost functions, which is similar to that in Beyer, Sethi and Taksar (1998).

Assumption 2. For each $n=0,1, \cdots, N-1$ and $i \in I$, we have

$$
c_{n}^{i} x+F_{n+1}\left(f_{n+1}\right)(i, x) \rightarrow+\infty \text {, as } x \rightarrow \infty
$$

Assumption 2 is not very restrictive in practice. It rules out unrealistic trivial cases of ordering an infinite amount, if $c_{n}^{i}=0$ and $f_{n}(i, x)=0$ for each $i$ and $n$. It is useful in proving the first part of Theorem 1 that follows. Moreover, in the proof of the $(s, S, p)$ policy in Theorem 3 of Section 4 , we do not need to impose a condition like (12) for $x \rightarrow-\infty$, as Assumption 3 in Chen and Simchi-Levi (2004). See Remark 4.4 in Sethi and Cheng (1997). 
From an analytical perspective, adding the price decision significantly complicates the traditional inventory control model. For ease of analysis, like most of the joint pricing and inventory control literature, we will assume that prices are continuous and restricted in a closed interval on the real line. As in the previous discussion of this section, the compact set of the feasible prices plays a critical role to generalize the existence and verification theorems in Beyer, Sethi and Taksar (1998) to our price-inventory Markovian demand model.

Now we are ready to state the two verification theorems as below. The proofs are similar to those in Beyer, Sethi and Taksar (1998). We omit the details here.

Theorem 1. The dynamic programming equations (10) and (11) define a sequence of functions in $B_{1}$. Moreover, for each $n=0,1, \cdots, N-1$ and $i \in I$, there exists a function $\hat{y}_{n}(i, x) \in B_{0}$, such that the supremum in (10) is attained at $y=\hat{y}_{n}(i, x)$ for any $x \in R$.

To solve the problem of maximizing $J_{0}(i, x ; U)$, we use $\hat{y}_{n}(i, x)$ of Theorem 1 to define

$$
\begin{aligned}
\hat{y}_{k} & =\hat{y}_{k}\left(i_{k}, \hat{x}_{k}\right), \quad k=0,1, \cdots, N-1 \text { with } i_{0}=i, \\
\hat{x}_{k+1} & =\hat{y}_{k}-\xi_{k}^{i_{k}}, \quad k=0,1, \cdots, N-1 \quad \text { with } \hat{x}_{0}=x, \\
\hat{u}_{k} & =\hat{y}_{k}-\hat{x}_{k}, \quad k=0,1, \cdots, N-1, \text { and } \\
\hat{p}_{k} & =p_{k}\left(i_{k}, \hat{y}_{k}\right), \quad k=0,1, \cdots, N-1 .
\end{aligned}
$$

We have the following verification theorem.

Theorem 2. The policy $\hat{U}=\left(\hat{u}_{0}, \hat{p}_{0}, \hat{u}_{1}, \hat{p}_{1}, \cdots, \hat{u}_{N-1}, \hat{p}_{N-1}\right)$ maximizes $J_{0}(i, x ; U)$ over the class $\mathcal{U}$ of all admissible decisions. Moreover,

$$
v_{0}(i, x)=\max _{U \in \mathcal{U}} J_{0}(i, x ; U)
$$




\section{Optimality of $(s, S, p)$ Policies}

To prove the optimality of an $(s, S, p)$ policy, we will use a similar approach as Chen and SimchiLevi (2004a) based on the concept of $K$-convexity introduced by Scarf (1960). See Propositions 4.1 and 4.2 in Sethi and Cheng (1997) for a summary of the properties of $K$-convex functions. We make the following assumption, which is required in the proof of Theorem 3 that follows.

Assumption 3. For $n=0,1, \cdots, N-1$ and $i \in I$, we have

$$
K_{n}^{i} \geq \bar{K}_{n+1}^{i}=\sum_{j=1}^{L} p_{i j} K_{n+1}^{j} \geq 0
$$

Condition (13) is a generalization of the similar conditions used in the standard models. It includes the cases of the constant ordering costs $\left(K_{n}^{i}=K, \forall i, t\right)$ and the non-increasing ordering $\operatorname{costs}\left(K_{n}^{i} \geq K_{n+1}^{j}, \forall i, j, n\right)$. See Remark 4.1 in Sethi and Cheng (1997) for a discussion.

Theorem 3. (a) For $i \in I, 0 \leq n \leq N-1, g_{n}\left(i, y, p_{n}(i, y)\right)$ is continuous in $y$, and $\lim _{y \rightarrow \infty} g_{n}\left(i, y, p_{n}(i, y)\right)=-\infty$.

(b) For $i \in I, 0 \leq n \leq N-1, g_{n}\left(i, y, p_{n}(i, y)\right)$ and $v_{n}(i, x)$ are $K_{n}^{i}$-concave.

(c) For $i \in I$, there exists a sequence of numbers $s_{n}^{i}, S_{n}^{i}, n \in 0,1, \cdots, N-1$, with $s_{n}^{i} \leq S_{n}^{i}$, such that the optimal replenishment policy is:

$$
\hat{u}_{n}(i, x)=\left(S_{n}^{i}-x\right) \delta\left(s_{n}^{i}-x\right)
$$

and the optimal selling price is:

$$
\hat{p}_{n}^{i}= \begin{cases}p_{n}\left(i, S_{n}^{i}\right), & \text { if } x_{n}<s_{n}^{i}, \\ p_{n}\left(i, x_{n}\right), & \text { if } x_{n} \geq s_{n}^{i}\end{cases}
$$

Proof. For part (a), the upper semicontinuity of $g_{n}\left(i, y, p_{n}(i, y)\right)$ was proven in Section 3 and 
the latter part follows from Assumption 2.

Next, we prove part (b) by induction. Notice that $v_{N}(i, x)$ is $K$-concave for any $K \geq 0$ since $v_{N}(i, x)=-f_{N}(i, x)$ and $f_{N}(i, x)$ is assumed to be convex in $x$, for $i \in I$. Now we assume that $v_{k+1}(i, x)$ is $K_{k+1}^{i}$-concave in $x$. By the definition of $F_{k+1}$ in (8) and Proposition 4.1 in Sethi and Cheng $(1997)$, it is easy to see that $F_{k+1}\left(v_{k+1}\right)(i, z)$ is $\bar{K}_{k+1}^{i}=\sum_{j=1}^{L} p_{i j} K_{k+1}^{j}$-concave in $z$. By Assumption 3, we know that $F_{k+1}\left(v_{k+1}\right)(i, z)$ is $K_{k}^{i}$-concave in $z$. For any $y<y^{\prime}$, let $z=y-D_{k}\left(i, p_{k}(i, y)\right)$ and $z^{\prime}=y^{\prime}-D_{k}\left(i, p_{k}\left(i, y^{\prime}\right)\right)$. Thus by Lemma 2 and Definition 2.2 in Chen and Simchi-Levi (2004), we have $z<z^{\prime}$, and for $\lambda \in[0,1]$,

$$
\left.F_{k+1}\left(v_{k+1}\right)\left(i,(1-\lambda) z+\lambda z^{\prime}\right)\right) \geq(1-\lambda) F_{k+1}\left(v_{k+1}\right)(i, z)+\lambda F_{k+1}\left(v_{k+1}\right)\left(i, z^{\prime}\right)-\lambda K_{k}^{i} .
$$

This is equivalent to

$$
\begin{aligned}
& E\left[v_{k+1}\left(i_{k+1},(1-\lambda)\left(y-D_{k}\left(i_{k}, p_{k}\left(i_{k}, y\right)\right)\right)+\lambda\left(y^{\prime}-D_{k}\left(i_{k}, p_{k}\left(i_{k}, y^{\prime}\right)\right)\right)-\beta_{k}^{i_{k}}\right) \mid i_{k}=i\right] \\
\geq & (1-\lambda) E\left[v_{k+1}\left(i_{k+1}, y-D_{k}\left(i_{k}, p_{k}\left(i_{k}, y\right)\right)-\beta_{k}^{i_{k}}\right) \mid i_{k}=i\right] \\
+ & \lambda E\left[v_{k+1}\left(i_{k+1}, y^{\prime}-D_{k}\left(i_{k}, p_{k}\left(i_{k}, y^{\prime}\right)\right)-\beta_{k}^{i_{k}}\right) \mid i_{k}=i\right]-\lambda K_{k}^{i} .
\end{aligned}
$$

In addition, the concavity of $R_{k}(i, p)$ and $-c_{k}^{i} y$ implies that

$$
\begin{aligned}
R_{k}\left(i,(1-\lambda) p_{k}(i, y)+\lambda p_{k}\left(i, y^{\prime}\right)\right) & \geq(1-\lambda) R_{k}\left(i, p_{k}(i, y)\right)+\lambda R_{k}\left(i, p_{k}\left(i, y^{\prime}\right)\right) \text { and } \\
-c_{k}^{i}\left((1-\lambda) y+\lambda y^{\prime}\right) & =(1-\lambda)\left(-c_{k}^{i} y\right)+\lambda\left(-c_{k}^{i} y^{\prime}\right)
\end{aligned}
$$

Adding (16), (17) and (18), and by (9), we get

$g_{k}\left(i,(1-\lambda) y+\lambda y^{\prime},(1-\lambda) p_{k}(i, y)+\lambda p_{k}\left(i, y^{\prime}\right)\right) \geq(1-\lambda) g_{k}\left(i, y, p_{k}(i, y)\right)+\lambda g_{k}\left(i, y^{\prime}, p_{k}\left(i, y^{\prime}\right)\right)-\lambda K_{k}^{i}$ 
Since $p_{k}\left(i,(1-\lambda) y+\lambda y^{\prime}\right)$ is the optimal price corresponding to $(1-\lambda) y+\lambda y^{\prime}$ in $(9)$, we have

$$
g_{k}\left(i,(1-\lambda) y+\lambda y^{\prime}, p_{k}\left(i,(1-\lambda) y+\lambda y^{\prime}\right)\right) \geq g_{k}\left(i,(1-\lambda) y+\lambda y^{\prime},(1-\lambda) p_{k}(i, y)+\lambda p_{k}\left(i, y^{\prime}\right)\right)
$$

Therefore,

$g_{k}\left(i,(1-\lambda) y+\lambda y^{\prime}, p_{k}\left(i,(1-\lambda) y+\lambda y^{\prime}\right)\right) \geq(1-\lambda) g_{k}\left(i, y, p_{k}(i, y)\right)+\lambda g_{k}\left(i, y^{\prime}, p_{k}\left(i, y^{\prime}\right)\right)-\lambda K_{k}^{i}$

by which we have proven that $g_{k}\left(i, y, p_{k}(i, y)\right)$ is a $K_{k}^{i}$-concave function of $y$.

Finally, we consider part (c). By Proposition 4.2 in Sethi and Cheng (1997) and equation (10), we can conclude that there exist $s_{k}^{i}<S_{k}^{i}$, such that $S_{k}^{i}$ maximizes $g_{k}\left(i, y, p_{k}(i, y)\right)$ and $s_{k}^{i}$ is the smallest value of $y$ for which $g_{k}\left(i, y, p_{k}(i, y)\right)=g_{k}\left(i, S_{k}^{i}, p_{k}\left(i, S_{k}^{i}\right)\right)-K_{k}^{i}$, and

$$
v_{k}(i, x)=-f_{k}(i, x)+c_{k}^{i} x+ \begin{cases}-K_{k}^{i}+g_{k}\left(i, S_{k}^{i}, p_{k}\left(i, S_{k}^{i}\right)\right), & \text { if } x<s_{k}^{i} \\ g_{k}\left(i, x, p_{k}(i, x)\right), & \text { if } x \geq s_{k}^{i}\end{cases}
$$

According to Theorem 2, the $(s, S, p)$-type policy defined in (14) and (15) is optimal.

Theorem 3 extends Theorem 3.1 in Chen and Simchi-Levi (2004a) to a Markov modulated demand model. While Theorem 3 is similar to Theorem 4.1 in Sethi and Cheng (1997) in terms of optimal ordering policies, adding the price decision complicates the induction proof. This now requires a similar result as Lemma 2 in Chen and Simchi-Levi (2004a), which is stated in the above proof. 


\section{Extensions}

\subsection{Optimality of $(s, S, p)$ Policy for the Emergency Order Case}

In Section 4, we assumed that unsatisfied demand in each period is fully backlogged. In practice, sometimes an emergency order could be placed and delivered at the end of the period when a stockout occurs. This ensures that a $100 \%$ service level is achieved in each period. In this section, we will prove the optimality of the $(s, S, p)$ policies when the retailer is allowed to use emergency orders.

The difference between the model with emergency orders and the one with full backlogging is that when the on hand inventory $x_{k}$ at the beginning of period $k$ and the amount $u_{k}$ delivered in period $k$ is less than the demand $\xi_{k}$, the portion $\xi_{k}-x_{k}-u_{k}$ could be satisfied immediately by an emergency order. In this case, the next period starts with zero on-hand inventory. Thus, the model dynamics over the interval $\langle n, N>$ can be expressed as:

$$
\left\{\begin{array}{l}
x_{k+1}=\left(x_{k}+u_{k}-\xi_{k}^{i_{k}}\right)^{+}, k=n, \cdots, N-1, \\
x_{n}=x \\
i_{k}, k=n, \cdots, N-1, \text { follows a Markov chain with transition matrix } P, \\
i_{n}=i .
\end{array}\right.
$$

For $k=0,1, \cdots, N-1$ and $i \in I$, we use the same function $c_{k}(i, u)=K_{k}^{i} \delta(u)+c_{k}^{i} u$ as the regular ordering cost. Let $h_{k}(i, x)$ be the surplus (holding) cost in period $k$ if $x_{k}=x$ and $i_{k}=i$. This is defined from $I \times R$ into $R^{+}$. We assume $-h_{k}(i, x) \in B_{2}, h_{k}(i, x)$ is convex and nondecreasing in $x$, and $h_{k}(i, x)=0, \forall x \leq 0$. Let $q_{k}(i, x)$ be the emergency ordering cost in period $k$ if $i_{k}=i$. This is also defined from $I \times R$ into $R^{+}$. We assume $-q_{k}(i, x) \in B_{2}, q_{k}(i, x)$ is convex and nonincreasing in $x$, and $q_{k}(i, x)=0, \forall x \geq 0$. Furthermore, we assume $q_{k}(i, x)$ is state-independent, i.e., $q_{k}(i, x)=q_{k}(j, x), \forall i, j \in I$. A commonly used emergency ordering cost 
function is the linear function $q_{k}(i, x)=\hat{c}_{k}^{i} x^{-}$, where $\hat{c}_{k}^{i}$ is the unit emergency ordering cost in period $k$ when $i_{k}=i$.

With cost functions defined, we can write the objective function over the interval $\langle n, N>$ as:

$$
J_{n}^{e}(i, x ; U)=E\left\{\sum_{k=n}^{N-1}\left[p_{k} \xi_{k}^{i_{k}}-c_{k}\left(i_{k}, u_{k}\right)-h_{k}\left(i_{k}, x_{k}\right)-q_{k}\left(i_{k}, x_{k}+u_{k}-\xi_{k}^{i_{k}}\right)\right]-h_{N}\left(i_{N}, x_{N}\right)\right\}
$$

where $U=\left(u_{n}, p_{n}, \cdots, u_{N-1}, p_{N-1}\right)$ is a history-dependent admissible decision for the problem.

Define the value function over $\left\langle n, N>\right.$ with $x_{n}=x$ and $i_{n}=i$ to be:

$$
v_{n}^{e}(i, x)=\sup _{U \in \mathcal{U}} J_{n}^{e}(i, x ; U)
$$

Then the dynamic equations for the value functions are:

$$
\begin{aligned}
v_{n}^{e}(i, x) & =-h_{n}(i, x)+c_{n}^{i} x+\sup _{y \geq x, \bar{p}_{k} \geq p \geq \underline{p}_{k}}\left[-K_{n}^{i} \delta(y-x)+g_{n}^{e}(i, y, p)\right], \text { and } \\
v_{N}^{e}(i, x) & =-h_{N}(i, x)
\end{aligned}
$$

where

$g_{n}^{e}(i, y, p)=R_{n}(i, p)-c_{n}^{i} y+E\left[-q_{n}\left(i, y-D_{n}(i, p)-\beta_{n}^{i}\right)+v_{n+1}^{e}\left(i_{n+1},\left(y-D_{n}\left(i_{n}, p\right)-\beta_{n}^{i_{n}}\right)^{+}\right) \mid i_{n}=i\right]$.

If $v_{n+1}^{e}(i, x)$ is upper semicontinuous in $x, E\left[v_{n+1}^{e}\left(i_{n+1},\left(y-D_{n}\left(i_{n}, p\right)-\beta_{n}^{i_{n}}\right)^{+}\right) \mid i_{n}=i\right]$ and $g_{n}^{e}(i, y, p)$ are upper semicontinuous in $(y, p)$. Thus for any $y \geq x$, there exists $p_{n}(i, y) \in\left[\underline{p}_{n}, \bar{p}_{n}\right]$, 
such that

$$
\begin{aligned}
v_{n}^{e}(i, x)= & -h_{n}(i, x)+c_{n}^{i} x+\sup _{y \geq x}\left[-K_{n}^{i} \delta(y-x)+g_{n}^{e}(i, y, p(i, y))\right], \\
g_{n}^{e}(i, y, p(i, y))= & \sup _{\bar{p}_{n} \geq p \geq \underline{p}_{n}} g_{n}^{e}(i, y, p) \\
= & R_{n}(i, p(i, y))-c_{n}^{i} y+E\left[-q_{n}\left(i, y-D_{n}(i, p(i, y))-\beta_{n}^{i}\right)\right. \\
& \left.+v_{n+1}^{e}\left(i_{n+1},\left(y-D_{n}\left(i_{n}, p\left(i_{n}, y\right)\right)-\beta_{n}^{i_{n}}\right)^{+}\right) \mid i_{n}=i\right] .
\end{aligned}
$$

It is easy to check that $g_{n}^{e}\left(i, y, p_{n}(i, y)\right)$ and $v_{n}^{e}(i, x)$ are upper semicontinuous. Thus we will have a similar existence theorem as Theorem 1 in the full backlog case.

Theorem 4. The dynamic programming equations (25) and (26) define a sequence of functions in $B_{2}$ in the emergency order case. Moreover, for each $n=0,1, \cdots, N-1$ and $i \in I$, there exists a function $\hat{y}_{n}(i, x) \in B_{0}$, such that the supremum in (25) is attained at $y=\hat{y}_{n}(i, x)$ for any $x \in R^{+}$.

Furthermore, we can prove that the Verification Theorem 2 still holds in the emergency order case and the optimality of the $(s, S, p)$ policy is established in the following theorem.

Theorem 5. Assume for each $n=0,1, \cdots, N-1$ and $i \in I$,

$$
\begin{aligned}
q_{n}^{\prime}-(i, 0) & \leq h_{n+1}^{\prime+}(i, 0)-c_{n+1}^{i}, \text { and } \\
c_{n}^{i} x & +F_{n+1}\left(h_{n+1}\right)(i, x) \rightarrow+\infty, \text { as } x \rightarrow \infty
\end{aligned}
$$

where $q_{n}^{\prime}-(i, 0)=\lim _{x \uparrow 0} \frac{\partial}{\partial x} q_{n}(i, x)$, and $h_{n+1}^{{ }^{+}}(i, 0)=\lim _{x \downarrow 0} \frac{\partial}{\partial x} h_{n+1}(i, x)$. Then an $(s, S, p)$ policy is optimal for the emergency order case.

Proof. To prove the optimality of an $(s, S, p)$ policy, since $g_{n}^{e}\left(i, y, p_{n}(i, y)\right)$ is upper semicontinuous in $y$ and $g_{n}^{e}\left(i, y, p_{n}(i, y)\right) \rightarrow-\infty$, as $y \rightarrow \infty$ (by Assumption (28)), we only need to 
show that $g_{n}^{e}\left(i, y, p_{n}(i, y)\right)$ is $K_{n}^{i}$-concave in $y$, by Proposition 4.2 in Sethi and Cheng (1997). This is done by induction. We assume that $v_{n+1}^{e}(i, x)$ is $K_{n+1}^{i}$-concave in $x$ and define:

$$
Q_{n}(i, z)=q_{n}(i, z)-v_{n+1}^{e}\left(i, z^{+}\right)
$$

Thus,

$$
\begin{aligned}
& E\left[-q_{n}\left(i, y-D_{n}(i, p(i, y))-\beta_{n}^{i}\right)+v_{n+1}^{e}\left(i_{n+1},\left(y-D_{n}\left(i_{n}, p\left(i_{n}, y\right)\right)-\beta_{n}^{i_{n}}\right)^{+}\right) \mid i_{n}=i\right] \\
= & E\left[-q_{n}\left(i_{n+1}, y-D_{n}\left(i_{n}, p\left(i_{n}, y\right)\right)-\beta_{n}^{i_{n}}\right)+v_{n+1}^{e}\left(i_{n+1},\left(y-D_{n}\left(i_{n}, p\left(i_{n}, y\right)\right)-\beta_{n}^{i_{n}}\right)^{+}\right) \mid i_{n}=i\right] \\
= & F_{n+1}\left(-Q_{n}\right)\left(i, y-D_{n}(i, p(i, y))\right), \text { and } \\
& g_{n}^{e}(i, y, p(i, y))=R_{n}\left(i, p_{n}(i, y)\right)-c_{n}^{i} y+F_{n+1}\left(-Q_{n}\right)\left(i, y-D_{n}(i, p(i, y))\right),
\end{aligned}
$$

where $F_{n+1}$ is defined as (8). Notice (30) is of the exact same form as (9) except with $v_{n+1}$ replaced by $-Q_{n}$. It is easy to check that $y-D_{n}\left(i, p_{n}(i, y)\right)$ is also nondecreasing in $y$ in the emergency order case. Thus if we could prove $-Q_{n}(i, z)$ is $K_{n+1}^{i}$-concave in $z$, then following the same argument as in the proof of Theorem 3, we could conclude that $g_{n}^{e}\left(i, y, p_{n}(i, y)\right)$ is $K_{n}^{i}$-concave in $y$.

To prove $Q_{n}(i, z)$ is $K_{n+1}^{i}$-convex in $z$, since $q_{n}(i, z)$ is convex and nonincreasing with $q_{n}(i, x)=0, \forall x \geq 0$, and $-v_{n+1}^{e}(i, z)$ is $K_{n+1}^{i}$-convex in $z$, by Proposition 3.1 in Cheng and Sethi (1999), it is sufficient to verify that $q_{n}^{\prime}-(i, 0) \leq-v_{n+1}^{e^{\prime}+}(i, 0)$. From the $K_{n+1}^{i}$-concavity of $v_{n+1}^{e}(i, z)$ and (25), we know there exist $s_{n+1}^{i}$ and $S_{n+1}^{i}$, with $0 \leq s_{n+1}^{i} \leq S_{n+1}^{i}$, such that

$v_{n+1}^{e}(i, x)=-h_{n+1}(i, x)+c_{n+1}^{i} x+ \begin{cases}-K_{n+1}^{i} \delta(y-x)+g_{n+1}^{e}\left(i, S_{n+1}^{i}, p_{n+1}\left(i, S_{n+1}^{i}\right)\right), & \text { if } x<s_{n+1}^{i}, \\ g_{n+1}^{e}\left(i, x, p_{n+1}(i, x)\right), & \text { if } x \geq s_{n+1}^{i} .\end{cases}$

Thus we have $-v_{n+1}^{e^{\prime}+}(i, 0)=h_{n+1}^{\prime+}(i, 0)-c_{n+1}^{i}$. By Assumption $(27)$, we have proven $q_{n}^{\prime}-(i, 0) \leq$ 
$-v_{n+1}^{e^{\prime}+}(i, 0)$. This completes the proof.

Assumption (27) means that the marginal emergency ordering cost in one period is larger than or equal to the regular unit ordering cost less the marginal inventory holding cost in any state of the next period. See Remark 3.2 in Cheng and Sethi (1999) for a discussion. To better understand this assumption, we consider a special case when all cost functions are state and time independent, $q_{n}(i, x) \equiv \bar{c} x^{-}, h_{n}(i, x) \equiv h x^{+}$and $c_{n}^{i} \equiv c$, where $\bar{c}, h, c>0$ are the unit emergency ordering, holding and regular ordering costs. Therefore Assumption (27) implies that $\bar{c} \geq c-h$, which is always true in practice since $\bar{c} \geq c$.

In the emergency order case, the demand in each period is satisfied immediately either by the current inventory on hand, or by regular or emergency orders. Therefore, the total expected revenue is the same as that in the full backlog case. It will be different if we assume that unsatisfied demand is totally lost, since the revenue in each period is equal to the price multiplied by the minimum of the demand and the inventory position. This would complicate the analysis as the expected revenue will no longer be concave. Therefore, it is possible that an $(s, S, p)$ policy is not optimal in the lost sales case. In the joint pricing and inventory control literature, several researchers have considered the independent additive demand model with lost sales in a finite horizon system, such as Chen, Ray and Song (2003) and Huh and Janakiraman (2005). They prove the optimality of an $(s, S, p)$ policy under the assumptions of stationary

parameters and a salvage value that is equal to the unit ordering cost. We tried to relax their stationary parameters assumption and generalize their results to the Markovian demand model with pricing and inventory decisions; however, the resulting analysis was intractable.

\subsection{An $(s, S, p)$ Model with Capacity and Service Constraints}

In Sethi and Cheng (1997), the authors consider the Markovian demand model with capacity and service level constraints. We generalize their analysis to our model with pricing decisions. 
Suppose the retailer has a capacity limit $D$, which is an upper bound for the inventory level in each period. Moreover, to guarantee a certain service level, we require that $\operatorname{Prob}\left(x_{k+1} \leq C\right) \leq$ $\alpha$, for $k=0,1, \cdots, N-1$, where $C$ is some pre-determined threshold and $0<\alpha<1$. Therefore when demand is in state $i$ at period $k$, for any price $p \in\left[\underline{p}_{k}, \bar{p}_{k}\right]$, the above condition means that $\operatorname{Prob}\left(x_{k}+u_{k}-D_{k}(i, p)-\beta_{k}^{i} \leq C\right) \leq \alpha$ and we have $x_{k}+u_{k} \geq D_{k}(i, p)+C+\Phi_{i, k}^{-1}(1-\alpha)$, where $\Phi_{i, k}(\cdot)$ is the cumulative probability function of $\beta_{k}^{i_{k}}$ given $i_{k}=i$, and $\Phi_{i, k}^{-1}(z)=\inf \left(x \mid \Phi_{i, k}(x) \geq\right.$ $z)$. Let $A_{k}^{i}=D_{k}\left(i, \underline{p}_{k}\right)+C+\Phi_{i, k}^{-1}(1-\alpha)$. Assuming any unsatisfied demand is backlogged, the dynamic programming equations are defined as (5), where:

$$
G_{n}(i, x)=\sup _{y \geq x, A_{n}^{i} \leq y \leq D, \bar{p}_{n} \geq p \geq \underline{p}_{n}}\left[-K_{n}^{i} \delta(y-x)+g_{n}(i, y, p)\right]
$$

and $g_{n}(i, y, p)$ is defined as in $(7)$.

Notice that both $y$ and $p$ are now defined in compact sets; therefore, we could implement the same sequential optimization procedure as in Section 3 and conclude that the dynamic programming equations (5) with (32) define a sequence of upper semicontinuous functions on $(-\infty, D]$. For any $x \in(-\infty, D]$, there exists a function $\hat{y}_{n}(i, x) \in B_{0}$ that attains the supremum in (5). Furthermore, we can check that Theorem 2 still holds and the optimality policy is of the $(s, S, p)$-type.

Theorem 6. There exist a sequence of numbers $s_{n}^{i}, S_{n}^{i}$ for $n=0,1, \cdots, N-1$ and $i \in I$, with $A_{n}^{i} \leq s_{n}^{i} \leq S_{n}^{i} \leq D$, such that the optimal feedback policy $\left(\hat{u}_{n}, \hat{p}_{n}\right)$ are of the same forms as defined in equations (14) and (15) in Theorem 3.

Proof. This follows by the similar induction as in the proof of Theorem 3 and properties of $K$-convex functions in Propositions 4.1 and 4.2 in Sethi and Cheng (1997). We omit the details. 


\section{Computation of Optimal $(s, S, p)$ Policies}

In this section, we discuss the computation of the state-dependent optimal $(s, S, p)$ policy for our basic model and present illustrative numerical examples.

Suppose for each $n, 0 \leq n \leq N-1$, and any state $i \in I,\left(s_{n}^{i}, S_{n}^{i}, p_{n}^{i}\right)$ are the parameters for the optimal policy. To guarantee global convergence of a computational algorithm, we need to find bounds for these parameters of the optimal policy. Following the similar method of Chen and Simchi-Levi (2004b), we can develop state-dependent bounds for the reorder point $s$ and order-up-to level $S$ as follows.

We assume that all the input parameters (demand processes, costs and revenue functions) are state dependent, but time independent. Thus we can omit the subindex of period $n$ from these parameters. Furthermore, we assume that the unit ordering cost is also state independent, which implies that $c_{n}^{i} \equiv c$, for all $n, 0 \leq n \leq N-1$, and $i \in I$. Also, time independence of $K_{n}^{i}$ and Assumption 3 imply that $K^{i} \geq \sum_{j=1}^{L} p_{i j} K^{j}, \forall i \in I$.

For each $i, i \in I$, and $\underline{p} \leq p \leq \bar{p}$, define:

$$
\begin{aligned}
H(i, y, p) & =-E f\left(i, y-D(i, p)-\beta^{i}\right)+R(i, p)-c D(i, p), \text { and } \\
H^{0}(i, y, p) & =H(i, y, p)+c D(i, p)-c y
\end{aligned}
$$

where functions $f(i, y)$ and $R(i, p)$ are the time-independent surplus cost and expected revenue defined in Section 2. 
Also, define:

$$
\begin{aligned}
\underline{S}^{i}= & \min _{\underline{p} \leq p \leq \bar{p}}\left\{\arg \max _{y} H(i, y, p), \arg \max _{y} H^{0}(i, y, p)\right\}, \\
\bar{s}^{i}= & \max _{\underline{p} \leq p \leq \bar{p}}\left\{\arg \max _{y} H(i, y, p)\right\}, \\
\underline{s}^{i}= & \max \left\{y \mid y \leq \underline{S}^{i}, H\left(i, \underline{S}^{i}, p\right) \geq H(i, y, p)+K^{i},\right. \text { and } \\
& \left.H^{0}\left(i, \underline{S}^{i}, y\right) \geq H^{0}(i, y, p)+K^{i}, \text { for all } p, \underline{p} \leq p \leq \bar{p}\right\}, \text { and } \\
\bar{S}^{i}= & \min \left\{y \mid y \geq \bar{s}^{i}, H\left(i, \bar{s}^{i}, p\right) \geq H(i, y, p)+K^{i}, \text { for all } p, \underline{p} \leq p \leq \bar{p}\right\} .
\end{aligned}
$$

We are now ready to state our bounds on $s_{n}^{i}$ and $S_{n}^{i}$.

Lemma 1. For every $n, 0 \leq n \leq N-1$ and every $i, 1 \leq i \leq L, s_{n}^{i} \in\left[\underline{s}^{i}, \bar{s}^{i}\right]$ and $S_{n}^{i} \in\left[\underline{S}^{i}, \bar{S}^{i}\right]$.

The proof of Lemma 1 is similar to those in Chen and Simchi-Levi (2004b); we omit its proof here.

We next use these bounds to construct numerical examples to better illustrate the ideas in this paper. We assume the riskless demand in state $i$ is a linear function of price $p: D(i, p)=$ $a^{i}-b^{i} p$, where $a^{i}$ represents the market size and $b^{i}$ represents customer price sensitivity. When demand is in state $i$, we assume the error term $\beta^{i}$ follows a discrete uniform distribution on interval $\left[-\lambda^{i}, \lambda^{i}\right]$, where $\lambda^{i}$ is a non-negative integer. Specifically, when demand is in state $i, \beta^{i}$ has the following probability mass function:

$$
P\left(\beta^{i}=k\right)= \begin{cases}\frac{1}{2 \lambda^{i}+1}, & \text { for } k \text { integer, } \quad-\lambda^{i} \leq k \leq \lambda^{i} \\ 0, & \text { otherwise. }\end{cases}
$$

We also assume the inventory holding/penalty cost function takes the following linear form in state $i$ :

$$
f(i, x)=h^{i} \max (0, x)+q^{i} \max (0,-x),
$$


where $h^{i}, q^{i}>0$ are the state-dependent unit inventory holding and penalty costs, respectively.

In the following examples, the entire horizon length is $N=24$ months and there are 3 demand states, i.e., $L=3$. We assume that all parameters take integer values and are time independent. The parameter specifications are:

$$
\begin{array}{r}
\left(a^{1}, a^{2}, a^{3}\right)=(60,75,30), \quad\left(b^{1}, b^{2}, b^{3}\right)=(2,3,1), \quad c=4, \quad K^{i} \equiv K=100 \\
\left(h^{1}, h^{2}, h^{3}\right)=(2,1,3), \quad\left(q^{1}, q^{2}, q^{3}\right)=(10,8,6), \quad\left(\lambda^{1}, \lambda^{2}, \lambda^{3}\right)=(20,15,5) .
\end{array}
$$

The demand state transition matrix can take the following forms:

$$
P_{1}=\left(\begin{array}{ccc}
0 & 1 & 0 \\
0 & 0 & 1 \\
1 & 0 & 0
\end{array}\right), P_{2}=\left(\begin{array}{ccc}
0 & 0.5 & 0.5 \\
0.5 & 0 & 0.5 \\
0.5 & 0.5 & 0
\end{array}\right) \text { and } P_{3}=\left(\begin{array}{ccc}
0.1 & 0.1 & 0.8 \\
0.6 & 0.2 & 0.2 \\
0.1 & 0.5 & 0.4
\end{array}\right)
$$

Notice $P_{1}$ represents a special case of the Markovian demand, which is called cyclic or seasonal demand.

The basic idea of our computation algorithm stems from the dynamic programming formulation (5) and equation (7). Suppose we start with zero inventory at the beginning of the horizon and $i_{0}=1$. Furthermore, we assume $f_{N}(i, x) \equiv 0$. Since our model has a finite horizon and $v_{N} \equiv 0$, one might consider using recursive method from period $N$. However, due to the complicated direct and indirect recursion among different functions, a recursive method suffers from poor efficiency because of a large number of function invocations. For example, for small size problems with $N=6$ and $L=3$, a recursive method cannot get results within three hours. To tackle this problem and make good use of the bounds developed at the beginning of this section, we use an iterative method. First, we figure out the biggest range of $y$ needed to compute functions $g_{n}(i, y, p)$ over the entire time horizon. Then we make sure that all the 
necessary $g_{n+1}$ function values are precomputed before each iteration step $n$. In this way, the algorithm has high efficiency and is able to solve problems with size $N, L \leq 100$ in seconds. See the Appendix for the detailed algorithm, which works for the Markovian demand model with any transition matrix and discretely and uniformly distributed random noise term.

We then use this algorithm to calculate the optimal $\left(s_{n}^{i}, S_{n}^{i}, p_{n}^{i}\right)$ policy for the above example using data in (40) with the cyclic demand corresponding to transition matrix $P_{1}$. As an approximation for the continuous range of the prices, we assume that price can only take discrete integer values with lower bound $\underline{p}=4$ and upper bound $\bar{p}=20$.

We report the optimal $\left(s_{n}^{i}, S_{n}^{i}\right)$ values in Table 1 for each pair of $(n, i), 0 \leq n \leq 23,1 \leq i \leq$ 3. An interesting observation is that under the assumption of time independent parameters, as time horizon $N$ becomes larger, the model tends to have stationary $\left(s^{i}, S^{i}\right)$ policy for each state $i$ and the optimal replenishment policy is cyclic. This is a similar result with Corollary 7.1 in Sethi and Cheng (1999), which states that a cyclic optimal policy exists for a cyclic demand model with an infinite horizon, when there are no pricing decisions.

\section{INSERT TABLE 1 ABOUT HERE.}

Next, using these numerical examples, we assess the benefits of dynamic pricing over fixed pricing across two different environments: Markovian demand and independent demands. The fixed price is chosen as the best price in $[\underline{p}, \bar{p}]$, at which the retailers optimal expected profit in the entire horizon is maximized. Table 2 summarizes the relative profit gains of dynamic pricing over fixed pricing in a Markovian demand case and three cases of independent demand. These

results are to be expected when there are no fixed costs of price changes, since the dynamic pricing strategy provides the retailer more flexibility to deal with demand changes. Note that the "Independent Demand $i$ " in Table 2 corresponds to the case when demand always stays in state $i$, for $i=1,2$ and 3 . Therefore, all parameters are time and state independent. Table 
2 also shows that it is always more beneficial to implement dynamic pricing in a Markovian demand setting than in the independent demand case. This confirms the intuition that it is always more effective to use price to hedge against demand uncertainty in a fluctuating demand environment.

\section{INSERT TABLE 2 ABOUT HERE.}

As another illustration, we conduct the sensitivity analysis on the fixed ordering cost $(K)$ by employing the same group of numerical examples. The result indicates that $K$ has a significant effect on the optimal replenishment policies and the benefit of dynamic pricing. In particular, as $K$ becomes larger, the optimal reorder point becomes lower and the optimal order-up-to level becomes higher. See Figure 1. This finding is intuitive and is similar to that found in Chen, Ray and Song (2003) for independent demand case. This means that when the retailers are charged a high fixed ordering cost every time an order is placed, they would rather wait until the inventory level drops low to reorder a large amount.

\section{INSERT FIGURE 1 ABOUT HERE.}

In Figure 2, we plot the relative profit gains of dynamic pricing over fixed pricing when $K$ changes from 0 to 200. It shows that when $K$ is increasing, the benefit of dynamic pricing over fixed pricing is increasing. This is true for both the Markovian demand and the independent demand cases. Furthermore, Figure 2 shows that when $K$ is high, the benefit of dynamic pricing in a Markovian demand setting is even more significant than the cases of independent

demand. This result is interesting. While a higher fixed ordering cost turns to have negative effects on the retailer's expected profit, dynamically adjusting the prices provides the retailer more benefit to manipulate the demand in a fluctuating demand environment.

\section{INSERT FIGURE 2 ABOUT HERE.}


Lastly, we consider the impact of demand uncertainty on the benefit of dynamic pricing over fixed pricing. Notice that in the above examples, we assume that the demand distribution is determined by a cyclic transition matrix $P_{1}$ defined in (41), which is a special class of Markovian demand. As long as the starting state is known, we immediately know the demand states in all periods over the entire time horizon. Since we allow for any arbitrary transition matrix in our model, we calculate the relative profit gains of dynamic pricing over fixed pricing when the transition matrix takes the forms of $P_{2}$ and $P_{3}$ defined in (41). Here, $P_{2}$ denotes the class of Markovian demand where demand will move to the other two states in the next period with equal probabilities and $P_{3}$ is an arbitrary transition matrix, since demand can take any state in the next period. Notice these classes of demand have more fluctuations than the cyclic demand represented by $P_{1}$ and the demand fluctuations of $P_{3}$ are greater than those of $P_{2}$. Table 3 shows that when the demand uncertainty increases (in terms of transition matrix), the expected profits will decrease (for both dynamic pricing and fixed pricing strategies), while the benefit of dynamic pricing over fixed pricing will increase.

\section{INSERT TABLE 3 ABOUT HERE.}

\section{Discussions and Conclusions}

In this paper, we extend the results in Chen and Simchi-Levi (2004a) to the Markovian demand model where the demand distribution at every period is determined by an exogenous Markov chain. We show that for additive demand functions, under the assumptions of backlogging and state-dependent cost functions, there exists an optimal Markov policy of the $(s, S, p)$-type which is also state dependent. We extend the basic model to the case of emergency orders and also incorporate capacity and service level constraints. We develop an algorithm to compute the optimal policy for a class of Markovian demand model with an arbitrary state transition 
matrix and a random noise term that follows a discrete uniform distribution. We consider the effects of fixed ordering cost and demand uncertainty on the benefits of dynamic pricing over fixed pricing through extensive numerical examples. The results show that it is more beneficial to implement the dynamic pricing strategy in a Markovian demand environment with a high fixed ordering cost or with high demand uncertainty.

Our paper assumes that inventory replenishment and pricing decisions are made simultaneously at the beginning of each period, before demand is realized. In practice, firms may adjust prices after they observe some demand information, which is referred to as the "responsive pricing" strategy in Chod and Rudi (2005). In their paper, they consider a single-period, twoproduct, and two-stage optimization problem, where the demand functions for both products take linear forms and the intercept terms are the only random variables following a bivariate normal distribution. Firms make the capacity decision at the first stage and the pricing decision at the second stage after the demand intercept terms materialize for both products. It might be interesting to extend our multi-period model to allow for responsive pricing. Another area for future work arises when we relax the assumption of perfect supply. This could occur when suppliers may not be able to deliver orders in full due to emergency factors, like power shutdowns and natural disasters. This introduces the concept of "Markovian supply" into our model. Parlar, Wang and Gerchak (1995) consider a periodic review inventory model with Markovian supply availability. They show that a state-dependent $(s, S)$ policy is optimal for an independent demand model under the assumption that the supplier can either deliver the orders in full amount or nothing. Extending their model to the case of Markovian demand with pricing decision could be another direction for future research. 


\section{APPENDIX}

\section{Algorithm to Compute the Optimal $(s, S, p)$ Policy}

Phase 1: Computation of Theoretical Bound

$i:=1$;

While $i \leq L$ do

begin

compute $\underline{S}^{i}, \bar{s}^{i}, \underline{s}^{i}, \bar{S}^{i}$ by equations (35), (36), (37) and (38);

$i:=i+1$

end.

Compute $\underline{y}$ and $\bar{y}$ by $\underline{y}:=\min _{i \in I} \underline{s}^{i}, \quad \bar{y}:=\max _{i \in I} \bar{S}^{i}$.

Phase 2: Computation of Optimal Policy

Set $v_{N}(i, x) \equiv 0$, and $n:=N-1$;

While $n \geq 0$ do

begin

$$
\begin{aligned}
& y_{1}:=\underline{y}-n\left(\max _{i \in I} a^{i}+\max _{i \in I} \lambda^{i}\right), \quad y_{2}:=\bar{y}+n \max _{i \in I} \lambda^{i} \\
& i:=1
\end{aligned}
$$

While $i \leq L$, do

begin

Step 2.1. Compute $g_{n}(i, y, p)$ for $y_{1} \leq y \leq y_{2}$, and $\underline{p} \leq p \leq \bar{p}$ by equation (7) with given or computed $v_{n+1}(i, x)$;

Step 2.2. For $y_{1} \leq y \leq y_{2}$, compute $p_{n}(i, y):=\min \left\{\arg \max _{\underline{p} \leq p \leq \bar{p}} g_{n}(i, y, p)\right\}$;

Step 2.3. $S_{n}^{i}:=\arg \max _{\underline{S}^{i} \leq y \leq \bar{S}^{i}} g_{n}\left(i, y, p_{n}(i, y)\right)$;

$$
s_{n}^{i}:=\min \left\{y: g_{n}\left(i, y, p_{n}(i, y)\right) \geq g_{n}\left(i, S_{n}^{i}, p_{n}\left(i, S_{n}^{i}\right)\right)-K^{i}, \underline{s}^{i} \leq y \leq S_{n}^{i}\right\}
$$

Step 2.4. If $x \geq s_{n}^{i}$, then

$$
v_{n}(i, x):=c x-f_{n}(i, x)+g_{n}\left(i, x, p_{n}(i, x)\right)
$$




$$
\begin{aligned}
& \hat{p}_{n}^{i}:=p_{n}(i, x) \\
& \text { else } \\
& v_{n}(i, x):=c x-f_{n}(i, x)-K^{i}+g_{n}\left(i, S_{n}^{i}, p_{n}\left(i, S_{n}^{i}\right)\right) \\
& \hat{p}_{n}^{i}:=p_{n}\left(i, S_{n}^{i}\right)
\end{aligned}
$$

Step 2.5. $i:=i+1$;

end;

$n:=n-1$;

end.

\section{References}

Beyer, D., S. Sethi, And M. TAKsar (1998): "Inventory Models with Markovian Demands and Cost Functions of Polynomial Growth," Journal of Optimization Theory and Applications, 98(2), 281-323.

Chan, L., Z. Shen, D. Simchi-Levi, and J. Swann (2004): "Coordination of Pricing and Inventory Decisions: A Survey and Classification," Handbook of Quantitative Supply Chain Analysis: Modeling in the E-business Era, Kluwer.

Chen, F. Y., S. Ray, and Y. Song (2003): "Optimal Pricing and Inventory Control Policy in Periodic-Review Systems with Fixed Ordering Cost and Lost Sales," Working paper, the Chinese University of Hong Kong.

Chen, H., O. Wu, and D. D. Yao (2004): "Optimal Pricing and Replenishment in a Single Product Inventory System," Working paper. 
Chen, X., And D. Simchi-Levi (2004a): "Coordinating Inventory Control and Pricing Strategies with Random Demand and Fixed Ordering Cost: The Finite Horizon Case," Operations Research, 52(6), 887-896.

(2004b): "Coordinating Inventory Control and Pricing Strategies with Random Demand and Fixed Ordering Cost: The Infinite Horizon Case," Mathematics of Operations Research, 29(3), 698-723.

Cheng, F., And S. P. Sethi (1999): "Optimality of State-Dependent (s,S) Policies in Inventory Models With Markov-Modulated Demand and Lost Sales," Production and Operations Management, 8(2), 183-192.

Chod, J., And N. Rudy (2005): "Resource Flexibility with Responsive Pricing," Operations Research, 53(3), 532-548.

Elmaghraby, W., and P. KeskinocaK (2003): "Dynamic Pricing in the Presence of Inventory Considerations: Research Overview, Current Practices and Future Directions," Management Science, 49(10), 1287-1309.

Federgruen, A., And A. Heching (1999): "Combined Pricing and Inventory Control Under Uncertainty," Operations Research, 47(3), 454-475.

Feng, Y., And F. Y. Chen (2003): "Joint Pricing and Inventory Control with Setup Costs and Demand Uncertainty," Working Paper.

(2004): "Optimality and Optimization of a Joint Pricing and Inventory-Control Policy for a Periodic-Review System," Working paper.

Gayon, J.-P., I. Degirmenci, F. Karaesmen, and E. Ormeci (2004): "Dynamic Pricing and Replenishment in a Production-Inventory System with Markov-Modulated Demand," Working paper. 
Huh, W., And G. Janakiraman (2005): "Optimality Results in Inventory-Pricing Control: An Alternative Approach," Working paper.

Kimes, S. (1989): "A Tool for Capacity-Constrained Service Firms," Journal of Operations Management, 8(4), 348-363.

LI, L. (1988): "A Stochastic Theory of the Firm," Mathematics of Operations Research, 13(3), $447-466$.

Parlar, M., Y. Wang, and Y. Gerchak (1995): "A Periodic Review Inventory Model with Markovian Supply Availability," International Journal of Production Economics, 42, $131-136$.

Petruzzi, N. C., And M. Dada (1999): "Pricing and the Newsvendor Model: A Review with Extensions," Operations Research, 47(2), 183-194.

Polatoglu, H., And I. Sahin (2000): "Optimal Procurement Policies Under PriceDependent Demand," International Journal of Production Economics, 65, 141-171.

ScArF, H. (1960): "The Optimality of (S,s) Policies in the Dynamic Inventory Problem," Mathematical Methods in the Social Sciences, edited by K. Arrow and S. Karlin and H. Scarf, Standford University Press, Standford, CA, 196-202.

Sethi, S. P., And F. Cheng (1997): "Optimality of $(s, S)$ Policies in Inventory Models with Markovian Demand," Operations Research, 45(6), 931-939.

Song, J. S., AND P. Zipkin (1993): "Inventory Control in a Fluctuating Demand Environment," Operations Research, 41(2), 351-370.

Veinott, A. (1966): "On the Optimality of (s,S) Inventory Policies: New Conditions and A New Proof," SIAM Journal on Applied Mathematics, 14(5), 1067-1083.

Whitin, T. M. (1955): "Inventory Control and Price Theory," Management Science, 2, 61-80. 
Table 1: Optimal $\left(s_{n}^{i}, S_{n}^{i}\right)$ values for $N=24, L=3$.

\begin{tabular}{|c|c|c|c|}
\hline & $\mathrm{i}=1$ & $\mathrm{i}=2$ & $\mathrm{i}=3$ \\
\hline $\mathrm{n}=0$ & $(15,67)$ & $(20,46)$ & $(-6,15)$ \\
\hline $\mathrm{n}=1$ & $(15,67)$ & $(20,46)$ & $(-6,15)$ \\
\hline $\mathrm{n}=2$ & $(15,67)$ & $(20,46)$ & $(-6,15)$ \\
\hline $\mathrm{n}=3$ & $(15,67)$ & $(20,46)$ & $(-6,15)$ \\
\hline $\mathrm{n}=4$ & $(15,67)$ & $(20,46)$ & $(-6,15)$ \\
\hline $\mathrm{n}=5$ & $(15,67)$ & $(20,46)$ & $(-6,15)$ \\
\hline $\mathrm{n}=6$ & $(15,67)$ & $(20,46)$ & $(-6,15)$ \\
\hline $\mathrm{n}=7$ & $(15,67)$ & $(20,46)$ & $(-6,15)$ \\
\hline $\mathrm{n}=8$ & $(15,67)$ & $(20,46)$ & $(-6,15)$ \\
\hline $\mathrm{n}=9$ & $(15,67)$ & $(20,46)$ & $(-6,15)$ \\
\hline $\mathrm{n}=10$ & $(15,67)$ & $(20,46)$ & $(-6,15)$ \\
\hline $\mathrm{n}=11$ & $(15,67)$ & $(20,46)$ & $(-6,15)$ \\
\hline $\mathrm{n}=12$ & $(15,67)$ & $(20,46)$ & $(-6,15)$ \\
\hline $\mathrm{n}=13$ & $(15,67)$ & $(20,46)$ & $(-6,15)$ \\
\hline $\mathrm{n}=14$ & $(15,67)$ & $(20,46)$ & $(-6,15)$ \\
\hline $\mathrm{n}=15$ & $(15,67)$ & $(20,46)$ & $(-6,15)$ \\
\hline $\mathrm{n}=16$ & $(15,67)$ & $(20,46)$ & $(-6,15)$ \\
\hline $\mathrm{n}=17$ & $(15,68)$ & $(20,46)$ & $(-6,15)$ \\
\hline $\mathrm{n}=18$ & $(15,67)$ & $(21,48)$ & $(-6,15)$ \\
\hline $\mathrm{n}=19$ & $(15,67)$ & $(20,45)$ & $(-6,15)$ \\
\hline $\mathrm{n}=20$ & $(14,71)$ & $(20,45)$ & $(-6,15)$ \\
\hline $\mathrm{n}=21$ & $(17,57)$ & $(24,54)$ & $(-6,15)$ \\
\hline $\mathrm{n}=22$ & $(18,46)$ & $(15,39)$ & $(2,26)$ \\
\hline $\mathrm{n}=23$ & $(-3,26)$ & $(-5,28)$ & $(-41,10)$ \\
\hline
\end{tabular}

Table 2: Expected profits in a Markovian demand case and three cases of independent demand.

\begin{tabular}{|c|c|c|c|}
\hline & Dynamic Pricing & Fixed Pricing & Relative Profit Gain \\
\hline Markovian Demand & 4720.66 & 4588.66 & $2.88 \%$ \\
\hline Independent Demand 1 & 5655.43 & 5607.44 & $0.856 \%$ \\
\hline Independent Demand 2 & 6099.14 & 6069.75 & $0.484 \%$ \\
\hline Independent Demand 3 & 2421.83 & 2386.53 & $1.48 \%$ \\
\hline
\end{tabular}

Table 3: Demand uncertainty impact on the benefit of dynamic pricing over fixed pricing. Case $i$ corresponds to demand transition matrix $P_{i}, i=1,2$ and 3 , as defined in (41).

\begin{tabular}{|l|c|c|c|}
\hline & Dynamic Pricing & Fixed Pricing & Relative Profit Gain \\
\hline Case 1 & 4720.66 & 4588.66 & $2.88 \%$ \\
\hline Case 2 & 4680.56 & 4540.53 & $3.08 \%$ \\
\hline Case 3 & 4396.09 & 4252.47 & $3.38 \%$ \\
\hline
\end{tabular}


Figure 1: Effects of fixed ordering cost $(K)$ on the optimal $\left(s^{i}, S^{i}\right)$ values. "** stands for the order-up-to levels, and "o" stands for the re-order points. As $K$ becomes larger, the values of $s^{i}$ are decreasing, and the values of $S^{i}$ are increasing. For $i=1,2$ and 3, we take the stationary values of $\left(s^{i}, S^{i}\right)$ indicated by Table 1 as representatives.
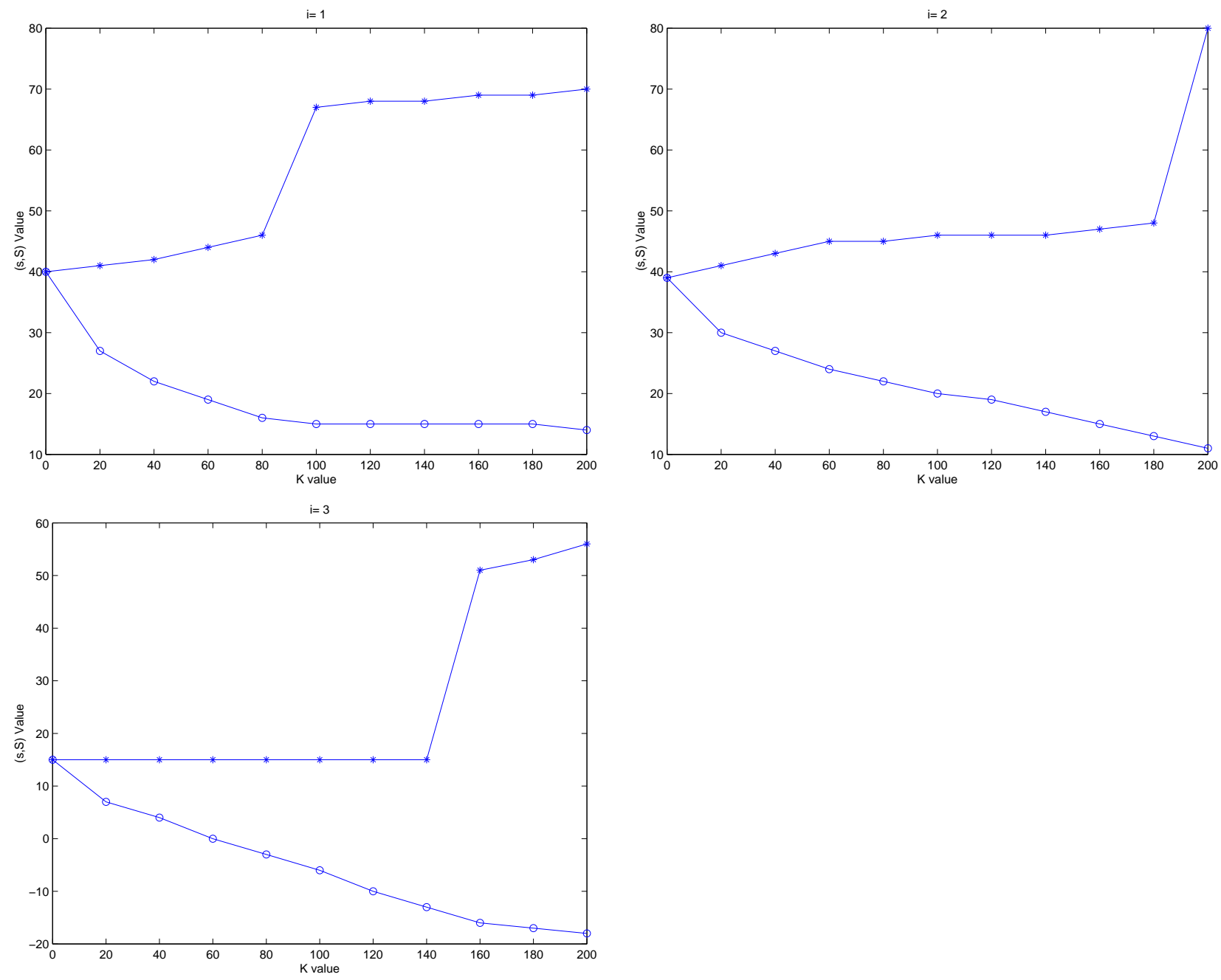
Figure 2: Effects of fixed ordering cost on the benefit (relative profit gain) of dynamic pricing over fixed pricing. "** stands for the Markovian demand case, and "o" stands for the independent demand case assuming demand is always in state $i$.
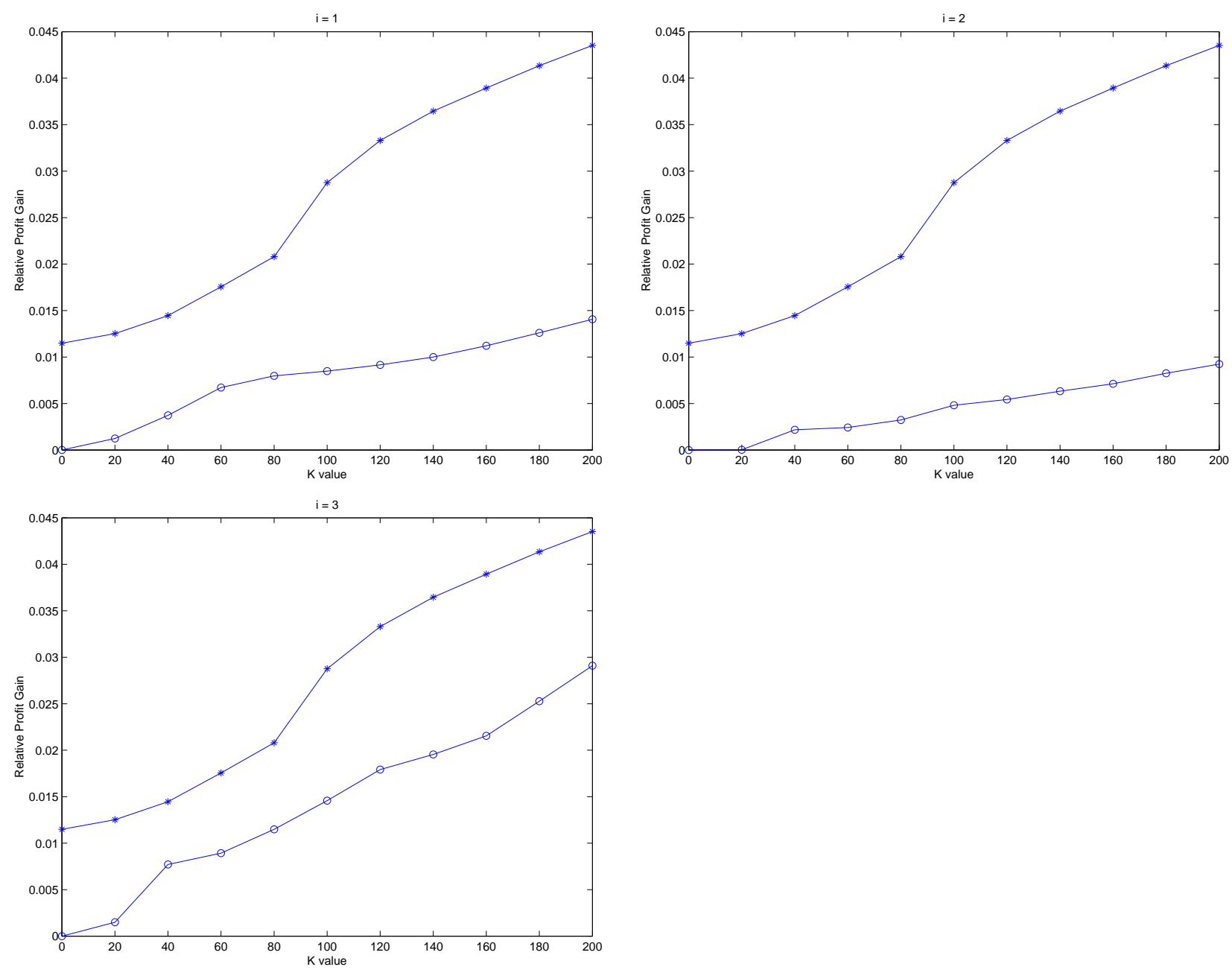